Résumés des conférences et travaux

\title{
Histoire et conscience historique des pays russes
}

\section{Pierre Gonneau}

\section{(2) OpenEdition Journals}

\section{Édition électronique}

URL : https://journals.openedition.org/ashp/3106

DOI : $10.4000 /$ ashp.3106

ISSN : 1969-6310

Éditeur

Publications de l'École Pratique des Hautes Études

\section{Édition imprimée}

Date de publication : 1 septembre 2019

Pagination : 298-303

ISSN : 0766-0677

\section{Référence électronique}

Pierre Gonneau, « Histoire et conscience historique des pays russes », Annuaire de l'École pratique des hautes études (EPHE), Section des sciences historiques et philologiques [En ligne], 150 | 2019, mis en ligne le 12 juin 2019, consulté le 06 juillet 2021. URL : http://journals.openedition.org/ashp/3106 ; DOI : https://doi.org/10.4000/ashp.3106 


\title{
HISTOIRE ET CONSCIENCE HISTORIQUE DES PAYS RUSSES
}

\author{
Directeur d'études : M. Pierre GonnEaU
}

Programme de l'année 2017-2018 : I. Le genre du Paterikon dans l'hagiographie slave orientale (XII ${ }^{e}-X V I^{e}$ s.). - - II. Chroniques et chroniques enluminées en Russie (XVe-XVI ${ }^{e}$ s.). III. Questions diverses.

\section{Le genre du Paterikon dans l'hagiographie slave orientale (XII $-X V I^{e}$ s.)}

Les séances ont été consacrées à la poursuite de la traduction du Paterikon des Grottes de Kiev (Kievo-Pečerskij Paterik), recueil consacré à la fondation de la plus importante abbaye de la Rus' médiévale et à ses premiers saints moines. Il a été achevé à Kiev dans le premier quart du XIII ${ }^{\mathrm{e}}$ siècle, sans doute vers 1223 . La traduction est faite à partir du texte établi pour la série Bibliothèque de la littérature de l'Ancienne Russie (Biblioteka literatury Drevnej Rusi), publiée sur papier et accessible en ligne sur le site de l'Institut de littérature russe de l'Académie des sciences de Russie ${ }^{1}$. Cette édition reprend la Deuxième rédaction du moine Cassien, datée de 1462, d'après un manuscrit de la fin $\mathrm{XV}^{\mathrm{e}}$-début $\mathrm{XVI}^{\mathrm{e}}$ siècle (Bibliothèque nationale de Russie à Moscou $=$ RGB fds. Rumjancev n ${ }^{\circ} 305$ ), complété pour les parties manquantes ou altérées par un témoin de la même époque (Moscou, Archives des actes anciens $=$ RGADA fds. Obolenskij, $n^{\circ}$ 69).

La structure du recueil est hybride : à la fois chronologique, logique et textologique. On sait que des notices relatant la fondation de l'abbaye des Grottes, les exploits ascétiques de ses premiers moines, mais aussi les tentations auxquelles ils succombent parfois, existaient dès le début du XII ${ }^{\mathrm{e}}$ siècle où elles ont trouvé leur place dans le Récit des temps passés (Povest'vremennyx let, v. 1110-1117), la plus ancienne compilation annalistique slave orientale. Mais le Paterikon proprement dit prend sa source dans la correspondance entre un ancien des Grottes, Simon, devenu évêque de Suzdal'-Vladimir (de 1214 à 1226) et le frère Polycarpe qui supporte mal de n'être pas déjà prélat. Simon exhorte cette ouaille indocile à prendre exemple sur les frères du passé. Cette épître est le «1 $14^{\mathrm{e}}$ discours 》 (slovo) de la Deuxième rédaction de Cassien. Elle est conservée là, comme une trace de l'échafaudage qui a permis la construction du recueil, contrairement à la Rédaction d'Arsène, évêque de Tver', datant de 1406, où les épîtres et les exhortations de Simon, placées à la fin de ses récits, ont été coupées. C'est pour cette raison que l'on préfère d'ordinaire la Deuxième rédaction de Cassien à celle d'Arsène. On trouve au passage dans ce $14^{\mathrm{e}}$ discours le mot smétie, ordure, impureté. $\mathrm{M}^{\mathrm{me}}$ Chwoles a attiré l'attention du séminaire sur une occurrence de ce terme dans un livre de souvenirs sur l'écrivain Isaac Babel. Babel connaissait le passage du Paterikon et à son époque le mot, sous la forme

1. Adresse $:$ http://lib.pushkinskijdom.ru/Default.aspx?tabid=2070. 
smit'e, était entré dans le parler odessite pour désigner un thé dans lequel les herbes flottent et ne sont pas bien déposées, une sorte de bouillasse ${ }^{2}$.

L'année précédente, le travail de traduction avait porté sur les discours 1 à 7 et 9 à 11 . Les discours 1 à 6 sont attribués à Simon. Ils évoquent la fondation, la construction, la décoration et la consécration de l'église de la Dormition, abbatiale du monastère des Grottes. Les travaux sont mentionnés par ailleurs dans le Récit des temps passés (s.a. du monde 6581 / 1073 à 6597 / 1089). Les récits mettent en évidence les liens étroits existant entre de grandes familles de guerriers varègues (i.e. scandinaves), proches des princes de Kiev (dont la lignée est également varègue) et le monastère. D'abord de rite latin, ils adoptent l'orthodoxie grâce à leur dévotion envers le « saint endroit » habité par la communauté des Grottes. Mais les six chapitres n'accordent pas moins d'importance aux liens spirituels et artistiques avec Constantinople. C'est de «Tsargrad » que viennent, invités par la Mère de Dieu elle-même, les architectes et les artistes qui vont donner à l'abbatiale sa splendeur. Le discours 7, dont l'auteur n'est pas connu, explique pourquoi le monastère des Grottes est ainsi appelé en présentant Antoine († 1072), premier frère et maître spirituel de la communauté, Théodose $(\dagger 1074)$, son organisateur, et le prince Izjaslav Jaroslavič qui règne sur Kiev (avec quelques interruptions) de 1054 à 1078. Les chapitres 8 à 11 sont attribués en général à l'hagiographe Nestor qui a composé la première Vie de Théodose vers 1080-1088. On en trouve une version abrégée (discours 8), suivie du récit sur la translation des reliques de Théodose en 1091 (discours 9), sur la décoration de sa tombe grâce aux dons de Georgij Simonovič, fils de celui qui avait fait construire l'église de la Dormition, v. 1129 (discours 10), et d'un Éloge prononcé le jour de la Saint-Théodose (discours 11).

Les traductions de cette année ont porté sur les discours 12 à 19. Les discours 12 et 13 , tous deux sans auteur connu, viennent clore le « cycle de Théodose ». Le 12 évoque rapidement les moines Damien, Jérémie et Mathieu, contemporains du premier abbé des Grottes. Le 13 raconte la visite de Niphôn, ancien des Grottes devenu évêque de Novgorod en 1131, au monastère en 1156, peu avant sa mort. Il a la grâce d'une vision au cours de laquelle Théodose lui promet qu'ils seront inséparables. De fait, il meurt le 18 avril et on l'enterre dans la grotte de Théodose. Les discours 15 à 23 sont l'œuvre de Simon et font suite à son épître à Polycarpe. Ils rapportent des exploits ascétiques rares ou des exempla mettant en garde contre tout laisser aller. Les discours 17 à 23 se terminent par un appel personnel à Polycarpe, soulignant la morale édifiante du récit.

\section{Chroniques et chroniques enluminées en Russie ( $\left.X V^{e}-X V I^{e} s.\right)$}

La publication récente de l'immense fonds iconographique que constitue la Chronique enluminée (Licevoj letopisnyj svod), assemblée sur la commande d'Ivan le Terrible entre 1568 et 1576, offre un terrain de recherche extrêmement riche. L'année précédente avait permis d'étudier le traitement du récit sur le concile de Ferrare-Florence (1439) qui aboutit, après le rejet de l'union des Églises par le grand-prince de Moscou et son clergé, à l'autocéphalie de l'Église russe (1448). On s'était aussi

2. A. N. Pirožkova, N. N. Jurgeneva, Vospominanija o Babele : sbornik, Moscou, 1989, p. 201. 
intéressé, dans le courant du récit annalistique à l'étrange épisode de la mort du prince Dmitrij Jur'evič le Bel, le 22 septembre 6949 / 1440. Cette année, un premier dossier a été consacré à Étienne de Perm', premier évêque missionnaire de l'Église russe auprès des populations de l'Oural (les Permiens, ou Zyrianes). La Chronique enluminée donne une version abrégée de l'ample Vie qui avait été dédiée au saint par Épiphane le Sage, au tout début du $\mathrm{Xv}^{\mathrm{e}}$ siècle ${ }^{3}$. Elle est insérée à la date de la mort du saint, le 26 avril 6904/1396 et comprend douze pages qui correspondent aux f. 571v à 577 du tome Osterman II (O.II) du Licevoj letopisnyj svod. Le texte correspondant avait déjà été publié dans l'édition de référence de la Chronique de Nikon ${ }^{4}$. Illustrant la mission d'Étienne, le f. O.II 573 montre la façon dont il brûle les idoles des Permiens : au milieu du brasier on distingue des habitations à toit pointu (sanctuaires?) et plusieurs formes qui semblent humaines. Le f. $574 \mathrm{v}$ évoque la gamme complète du paganisme local: «c'était merveille à voir en ce pays où l'on pratiquait l'idolâtrie et la démonolâtrie depuis le commencement du monde, en priant les idoles, le soleil, le feu, l'eau, les pierres, les arbres, les bœufs, les chèvres, les sorciers, les mages et la vieille d'or » (bě čjudno viděti v" zemli toj, iděže preže bě idolosluženie i běsom" ugoženie ot" načala miru, moljaščesja idolom", solncu, ognju, vodě, kameniju, dreviju, volom", kozam", i kudesnikom", i volxvom", i zolotoj babě). L'énumération a, certes, un caractère conventionnel, d'autant plus qu'Épiphane aime à tresser une « guirlande verbale » (pletenie sloves) dans laquelle il enserre son lecteur. Toutefois, on ne peut manquer de relever la zolotaja baba qui arrive en fin de liste et au bas de la page. L'enluminure ne nous éclaire pas sur son compte. Alors que l'on voit bien sur le feuillet un soleil rouge, un bœuf et une chèvre, un rocher, du feu, une rivière, des arbres auprès desquels se tiennent des hommes en posture orante, tandis qu'un démon noir, hirsute et nu, occupe l'angle inférieur droit de l'image, rien ne représente l'ultime idole. Sa réputation est pourtant bien établie, puisque même les observateurs étrangers en ont entendu parler, comme Sigismond von Herberstein qui se rend en Russie en 1517-1518 et en 1525. Il est plutôt disert à son sujet, bien qu'il ne l'ait pas vue lui-même : "Slata baba [de zlataja, forme concurrente de zolotaja], c'est-à-dire la « vieille d'or» (Aurea anus / Guldene Vetl oder Anfrau), est une idole située dans la province d'Obdora, sur les bouches de l'Ob, mais au-delà du fleuve (...), cette « vieille d'or » serait une statue faite à la ressemblance d'une vieille femme, tenant un enfant sur son sein; on dit même qu'il est possible de discerner à ses côtés un autre enfant, probablement son petit-fils; enfin elle aurait déposé là des instruments de musique qui rendent en permanence un son comparable à celui d'une trompette. S'il en est bien ainsi, ce son est produit, à mon avis, par le souffle puissant et incessant des vents qui

3. Voir aussi A. Vaillant, «Notes sur la Vie d'Étienne de Perm’», Revue des études slaves, 45 (1966), p. 33-37.

4. Licevoj letopisnyj svod XVI veka, Russkaja letopisnaja istorija. Kniga 11, 1393-1402, Moscou, 2010, p. 334-345 [l'édition que l'on trouve en ligne donne une indication erronée pour le Kniga 11, Rus' 1400-1410 ot V.X. et une pagination légèrement différente, p. 336-347]; Polnoe sobranie russkix letopisej [ci après PSRL], t. 11, Moscou, 1965, p. 164-166. Le manuscrit de la Chronique enluminée d'Ivan le Terrible reprend le texte de la Chronique de Nikon pour les parties concernant l'histoire de la Rus' et de la Moscovie. 
s'engouffrent dans ces instruments $»^{5}$. Herberstein, repris par Alessandro Guagnini (1578) ou Giles Fletcher (1591) situe l'idole sur l'Ob, alors que le Polonais Maciej de Miechow (1517) la plaçait « outre-Vjatka». Certains en ont conclu que l'idole était mobile, ou a été déplacée vers l'est, au fur et à mesure de l'avancée du christianisme $^{6}$. Baba a plusieurs significations : grand-mère, femme en général, en particulier épouse ou veuve, sage-femme, nounou, mais aussi, en mauvaise part, bonne femme, sorcière, magicienne. Pour compléter le récit de la Chronique enluminée, ont été lus les passages de la Vie d'Étienne de Perm' décrivant sa lutte contre le sorcier-centenier de la contrée (Pam sotnik), l'un des témoignages anciens sur le chamanisme dans la région de l'Oural. Les icônes biographiques d'Étienne ont été mises à contribution, notamment celle de Sol'-Vyčegodsk. La tardive Chronique de la Vyčegda et du Vym, considérée comme authentique par le spécialiste B. N. Florja, ressemble cependant à la Chronique de Joachim inventée par l'historien V. N. Tatiščev au début du $\mathrm{XVIII}^{\mathrm{e}}$ siècle $^{7}$.

En préparation d'une conférence internationale sur la Hongrie dans les sources narratives des $\mathrm{X}^{\mathrm{e}}-\mathrm{XVII}^{\mathrm{e}}$ siècles, il a été procédé à la recension des mentions de la Hongrie dans le Licevoj letopisnyj svod. Elles sont nettement réparties entre un premier lot concernant l'époque de la Rus' de Kiev (26 entrées couvrant les années 1119 à 1248 ) et un second concernant la période moscovite (19 entrées de 1386 à 1503 et deux entrées isolées, en 1543 et 1557). Pour la période moscovite, on privilégie nettement les relations entre Ivan III et Matthias Corvin, puis leur relâchement après la mort du roi. Pour la période ancienne, la Hongrie et est représentée avant tout dans un contexte militaire, comme partie prenante dans les guerres concernant la Galicie et la Volynie, refuge des vaincus, force militaire souvent décisive dans les batailles, faiseuse de princes. Un épisode héroïcomique attire l'attention. En 6659 / 1151, le prince Mstislav Izjaslavič s'appuie sur des troupes d'élites hongroises, mais il est cruellement déçu parce qu'un faux allié s'arrange pour faire boire excessivement les Magyars qui s'avèrent incapables de combattre lors d'une attaque-surprise. Or, la Chronique enluminée rapporte un épisode typologiquement identique, s.a. 6885 / 1377. Il est connu comme l'Histoire de la bataille de la P'jana, occasion d'un jeu de mots avec le nom de la rivière (litt. La Soule) ${ }^{8}$. Le contexte religieux suscite aussi

5. La Moscovie du XVIe siècle vue par un ambassadeur occidental, R. Delort (éd.), Paris, Herberstein, 1965, p. 140-141. S. Herberstein, Zapiski o Moskovii v dvux tomax, Moscou, 2000, t. 1, p. 372, où l'on trouve en parallèle le texte latin (1556) et allemand (1557) d'Herberstein, ainsi que leur traduction russe.

6. S. Mund, Orbis Russiarum : genèse et développement de la représentation du monde « russe » en Occident à la Renaissance, Genève, 2003, p. 250-251, 254, 259, 300-301 (avec gravure de la Slata baba, d'après la Cosmographie universelle de A. Thevet, 1575), 444, 454; I. Šlionskaja, «Zolotaja baba: glavnyj idol na territorii Drevnej Rusi », 13/10/2017 http://russian7.ru/post/zolotaya-baba-glavnyyidol-na-territor/.

7. «Vyčegodsko-Vymskaja (Misaio-Evtixievskaja) letopis’», dans Dokumenty po istorii Komi, P. G. Doronin (éd.), Syktyvkar, 1958 (Istoriko-filologičeskij sbornik Komi filiala AN SSSR 4), p. $257-$ 264.

8. PSRL.11.27-28. F. S. Kapica, « Letopisnaja 'Povest' o bitve na reke P'jane' », dans Drevnerusskaja literatura: tema Zapada v XIII-XV vv i povestvovatel’noe tvorčestvo, M., 2002, p. 100-103; V. N. Rusinov, «K ob"jasneniju dvux mest v tekse letopisnogo rasskaza o P'janskom poboišče 1377 g. », dans Pro- 
d'autres associations internes au Licevoj letopisnyj svod, plus surprenantes. Les Hongrois sont réputés n'avoir succombé à « l'hérésie latine » que par défaut, parce qu'ils ne disposaient pas de livres dans leur langue. Or, pareille circonstance est évoquée à propos des Bretons, ancêtres des Anglais, dans le long récit sur le concile de Florence (voir rapport de l'année précédente). Mais le cas des Hongrois est développé davantage encore, puisque l'on affirme que leur roi serait revenu à l'orthodoxie, en cachette de son peuple, grâce à la prédication de saint Sava, premier archevêque de Serbie (s.a. 6712 / 1204). Le nom du roi n'est pas donné dans cet épisode, mais le chroniqueur affirme que le même roi, appelé Vlaslov, puis Vladislav sous son nom orthodoxe, a vaincu et tué de sa main le khan tatar Batu en 6756/1248. La Hongrie a eu, de fait, plusieurs rois Ladislas (László), mais lors des invasions tatares elle était sous le sceptre de Bela IV (1235-1270), ce que la Chronique enluminée indique correctement dans d'autres passages. En fait, le texte de l'année 6756 est un récit hagiographico-politique autonome, connu sous le nom de Dit sur la mise à mort du maléfique tsar Batu (Slovo ob ubienii zločestivogo carja Batyja) ${ }^{9}$. Dans le contexte russe, il vient compléter le récit du martyre du prince Mixail de Černigov à la Horde d'Or, en punissant Batu qui a, effectivement, fait mettre à mort Mixail en 1246. On attribue la composition de ce diptyque à Pacôme le Serbe, un hagiographe venu des Balkans, et elle daterait de $c a 1479$. Le plus intéressant pour nous est que Pacôme s'est livré à un emprunt dans la tradition hagiographique hongroise. Les spécialistes russes sont très vagues sur sa source, alors qu'elle peut être établie. Il s'agit d'un épisode de la légende de saint Ladislas I ${ }^{\text {er }}$, roi de Hongrie de 1077 à 1095. Il est décrit précisément dans la Chronica Hungarorum, ou Chronicon pictum (dit aussi Chronique enluminée de Vienne), précieux manuscrit enluminé (achevé en 1370-1373) ${ }^{10}$. Plusieurs églises situées sur des territoires ayant fait partie du royaume de Hongrie (jusqu'en Roumanie et Slovaquie actuelles) présentent des fresques où l'on voit Ladislas vaincre et tuer un cavalier couman qui avait enlevé une puella speciosa, tout comme le Vladislav du Dit sur la mise à mort de Batu tue le Tatar fugitif, mais aussi sa propre sœur qui a été enlevé par le khan. On note que sur les fresques hongroises la jeune fille prend une part active au combat, ce qui témoigne d'une variante dans le récit par rapport aux textes connus.

\section{Questions diverses}

La séance du 8 novembre 2017 a été consacrée à la présentation de l'édition fac-similé de l’Évangéliaire de Reims, réalisée sous la direction du P. Alexandre

blemy proisxoždenija i bytovanija pamjatnikov drevnerusskoj pis'mennosti i literatury, Nižnij Novgorod, 2009, p. 101-142.

9. PSRL. 10.135-136; C. J. Halperin, « The Defeat and Death of Batu », Russian History, 10.1 (1983), p. 50-65; O. M. Ul'janov, « Smert' Batyja (k voprosu o dostovernosti letopisnogo soobščenija o gibeli v Vengrii zolotoordynskogo xana Batyja), Sbornik Russkogo istoričeskogo obščestva, 1 (1999), p. 157 170 ; A. A. Gorskij, « Povest' ob ubienii Batyja i russkaja literaturA 70-x godov XV veka », Srednevekovaja Rus', 3 (2001), p. 191-221; A. B. Straxov, «O duxovno-političeskix smyslax Slova ob ubienii zločestivogo carja Batyja”, Istoričeskoe obrazovanie, 2.2 (2014), p. 100-105.

10. Texte: Chronicon Budense, J. Podhradczky (éd.), Buda, 1838, p. 127-129. Version numérique du manuscrit enluminé http://konyv-e.hu/pdf/Chronica_Picta.pdf, voir p. 72-74. 
Siniakov, avec le concours de ce dernier et avec pour discutante Valérie Géronimi, élève diplômée de l'EPHE. La discussion a porté sur l'histoire controversée de la genèse, de la circulation et des publications de ce manuscrit double (glagolitique et cyrillique). Outre l'édition du P. Siniakov, a été largement utilisé le livre d'Olga B. Strakhova, Glagoličeskaja čast'Rejsmkogo Evangelija: istorija, jazyk, tekst, Cambridge (MA), 2014 (Palaeoslavica. Supplementum 3) que son auteur avait envoyé au directeur d'études. V. Géronimi a depuis publié un article faisant le point sur la question : "L'Évangéliaire slavon de Reims : mythes, (re)découverte historique et perspectives », Revue des études slaves, 89.1-2 (2018), p. 133-142.

Le 15 novembre, Alexey V. Krajkovskij, membre du Laboratoire d'histoire écologique et technologique de l'École des hautes études économiques de Saint-Pétersbourg (Высшая школа экономики, Лаборатория экологической и технологической истории) et chercheur invité par l'ENS Paris, a fait, dans le cadre du séminaire, une communication intitulée «"Like a Groom in his wedding day" - the Gulf of Finland as Metropolitan bay of Russia. The Baltic Sea in the environmental, technological and cultural history of St. Petersburg ». Cette présentation, illustrée d'images des XVIII ${ }^{\mathrm{e}}$ et $\mathrm{XIX}^{\mathrm{e}}$ siècle, montrait les différents rôles joués par le golfe de Finlande dans la vie de la capitale impériale russe, Saint-Pétersbourg. D'abord force naturelle dangereuse qu'il importe de maîtriser (les crues historiques de 1724 et 1824 laissent un souvenir douloureux), il devient peu à peu un havre domestiqué, propice aux excursions mondaines, bordé de palais et de villas, un morceau de terre aux confins de l'Empire qui contribue activement à son européanisation. Renouvelant les analogies traditionnelles avec Amsterdam, Venise ou Londres, A. Kraykovsky considère Saint-Pétersbourg et sa façade baltique à l'époque tsariste comme l'équivalent de Hong-Kong et de Macao au XIX ${ }^{\mathrm{e}}$ et au XX $\mathrm{XX}^{\mathrm{e}}$ siècle en tant que porte d'entrée de l'influence européenne dans une autre grand empire traditionnellement fermé.

Dans le cadre de la plateforme Multipal, tutoriels de paléographie, développée par l'EPHE http://multipal.fr/fr, M. Victor Smirnov, doctorant du directeur d'études, a procédé à la publication de quatre chartes russes médiévales provenant des anciennes archives de la municipalité de Riga. Les notices de ces documents avaient été préparées par le directeur d'études et $\mathrm{M}$. Aleksandrs Ivanovs, professeur à l'université de Daugavpils (Lettonie), directeur d'études invité en 2016. 